Chapter 4

\title{
Potential Future Ranges of Tree Species in the Alps
}

\author{
Niklaus E. Zimmermann, Robert Jandl, \\ Marc Hanewinkel, Georges Kunstler, \\ Christian Kölling, Patrizia Gasparini, \\ Andrej Breznikar, Eliane S. Meier, Signe Normand, \\ Ulrich Ulmer, Thomas Gschwandtner, Holger Veit, \\ Maria Naumann, Wolfgang Falk, Karl Mellert, \\ Maria Rizzo, Mitja Skudnik and Achilleas Psomas
}

Additional information is available at the end of the chapter

http://dx.doi.org/10.5772/56279

\section{Introduction}

Climate is one of the major drivers of plant and tree distribution, while soil variables or interspecific competition often are considered to primarily drive their local abundance. While the later is partly debated (Meier et al. 2010, 2011, 2012), the climate constraint to species ranges is generally accepted (Woodward 1987). The debate on climate change impacts on biodiversity and ecosystems is therefore specifically relevant to long-lived plants such as trees or shrubs, as these take many years to reach maturity and fecundity, and given their sessile growth strategy they are specifically vulnerable to rapid changes in climatic conditions. Also, forest management typically encompasses many decades, partly even reaching to the end of the $21^{\text {st }}$ century, which highlights the challenge to manage such organisms successfully at such long planning horizons. This calls for careful and adaptive management strategies and for a good understanding of the uncertainties related to the expected changes and its impacts on trees and forest ecosystems.

Many approaches exist to project the impact of climate change on trees and forests. Yet, most of the approaches either can be applied only to comparably small regions, to few species only, or they need to be run at very coarse spatial resolutions in order to enable coverage of larger spatial extents. We distinguish the following five basic approaches of which we list major advantages and disadvantages. We then explain how we have used the last of these five 
approaches to project how species might respond in their habitat preference at the scale of the European Alps in response to projected climate change. Other approaches exist and often they glean from the five approaches described below:

1. Biogeochemistry-driven dynamic vegetation models (DVMs) are considered mechanistic with regards to physiological processes, and therefore represent growth and the fluxes of water, carbon (Sitch et al. 2003), and - in some cases - nitrogen cycling (Thornton et al. 2002). They are driven by input parameters such as daily precipitation, temperature, vapor pressure, or global radiation, and they model the water fluxes based on climatic input, on soil properties, and on biogeochemical processes of the vegetation. The behavior of vegetation is described in a larger set of parameters such as the C:N ratio of leaves, roots, stemwood, of values that decribe photosynthetic or hydraulic properties. Such parameters are time consuming to measure, and therefore such models simulate the behavior of plant functional types (or biomes) rather than individual species. Demography or structural details of the forest stand, interspecific competition or stochastic disturbances are handled less well (for an exception, see Hickler et al. 2012), and these models are most often operated at very coarse spatial resolution such as $10^{\prime}$ to $0.5^{\circ} \mathrm{Lat} / \mathrm{Lon}$ cells, and are therefore not easily applicable to forest management. This model family does usually not distinguish individual trees or cohorts, but rather biomass pools and translocation of carbon and energy between pools. They are useful when investigating coarse scale fluxes of carbon, water and nitrogen, and in studying the re-distribution of coarse biomes such as deciduous broadleaf, evergreen needleleaf, or C3 grassland vegetation belts in response to climate change.

2. Population dynamic models of vegetation such as the forest gap models (Bugmann 2001) represent a model family that is highly suited for forest management applications at regional spatial scale. These models are process-oriented - although they lack the physiological processes of DVMs - and include significant demographic and structural details. They simulate the fate of individual trees or cohorts, and they simulate both intra- and interspecies competition within a forest stand. Many of these models are not spatially explicit, and thus are operated at a single stand level. Few versions exist that are spatially explicit, and thus allow for regional assessment of management and climate change impacts. The model LandClim (Schumacher et al. 2006) is an example of such a spatially explicit forest dynamics model, while TreeMig (Lischke et al. 2006) is another. The latter additionally includes seed maturity, seed dispersal, and seed bank processes, and models the full regeneration process that is lacking in most other models. It therefore allows for simulating the natural migration of trees in a landscape following climate change. The model can be run at comparably fine spatial resolution, but regions such as the whole Alps or even all of Europe cannot easily be simulated due to computational demands. While forest gap models were the first such model type, new versions of demographic vegetation models that include other (than tree) plant functional types are currently being developed. While stand structural details are often well simulated in such population dynamic models, NPP, LAI and other ecosystem properties are less well simulated. Although, some models exist that are specifically oriented towards modelling such physiological details in forest stand 
models. Such models can be applied to any forest, but due to the comparably high level of demographic detail, it is important to provide the necessary stand structural data as input to such models, in order to render them useful for forest management applications. Such input can be derived from forest inventories, although such information is not available yet at high spatial resolution. More research with regards to calibrating stand structure data from remotely sensed data sources is required to fill this gap.

3. Demographic range models (DRMs; Schurr et al. 2012) operate in a similar fashion as population dynamic models in that they also simulate the demography of species. They do so in a spatially explicit manner, but they usually lack inter-species competition, and only model one species at a time. This model type is thus best suited for treating dominant species. Habitat suitability is taken from species distribution models (see the fifth model family below), while demographic processes and spatial migration are modelled explicitly. These models can be applied to larger spatial scales, but are moderately demanding to calibrate new species, as the model is very data hungry. It requires information on seed dynamics, on seed dispersal distances, and on the spatial population behavior. They can be seen as simplified versions of the second model family, although they are more data driven in their calibration, while forest gap models are more based on conceptual first principles.

4. Phenological (partly physiological) models such as PhenoFit (Chuine \& Beaubien 2001, Chuine 2010) and conceptually similar models (Huey et al. 2012) attempt to calibrate those processes that directly affect - and moreover - constrain the demographic processes and life history, such as leaf unfolding, seed maturity, juvenile survival, etc. Such models therefore attempt to calibrate significant elements of the fundamental niche (Hutchinson 1957), while inter-species competition or demographic processes are not modelled. This model family can easily be applied to large spatial extents, but the calibration is very demanding for each species, so that only very few tree species have been calibrated to date.

5. Species distribution models (SDMs) are a simple but very efficient statistics-based method to map the spatial range of species and to project climate change impacts on species ranges (Zimmermann et al. 2010). The method is well matured from the statistical and conceptual end (Guisan \& Thuiller 2005, Elith et al. 2006), and it is used for many different purposes including conservation management, theory testing in biogeography and ecology, species management, climate change impact assessment (Guisan \& Zimmermann 2000). The method is based on calibrating statistical relationships between the observed spatial distribution of a species and climate and other spatial predictor variables that may explain this distribution. Sampling design is therefore very important, and the fitted spatial patterns represent the realized, not the fundamental niche. The method does not encompass any processes or details on transient responses following change, and no structural information is provided. Therefore, the method simply - but efficiently - provides an assessment of the suitability of any region for a species under current or projected future climate, and under the assumption that roughly the same species are available as potential competitors. SDMs are thus often used to assess, whether a given species has a future in a specific region or not, while the question whether it can reach a certain region and how 
long it would take a species to get there are not handled by this method. SDMs therefore are best suited to assess habitat suitability and whether certain management options regarding species preference/selection are likely sustainable in the long run.

In the following, we present SDM simulations, often also termed climate envelope models (CEM), for major tree species of the European Alps in order to assess what the consequences of climate change on the habitat suitability of these tree species is. We used presence/absence information from forest inventories of France (Alps only), Northern Italy ${ }^{1}$, Austria, Southern Germany Slovenia and Switzerland in order to build a database of tree species presences and absences across the Alps. We compiled data for ca. 50 tree species at a total of $>80^{\prime} 000$ inventory plots, although some countries did not distinguish all species at the same taxonomic level (some countries did e.g. not distinguish the different oaks or maples). We then compiled a series of climate maps under current and potential future climate from downscaled RCM models for future climates. We also compiled some topographic variables that may add to influence the spatial patterns of trees.

We finally used the following variables as predictors of species distribution in our models: (1) degree days with a $5.56^{\circ} \mathrm{C}$ threshold, (2) temperature seasonality (standard deviation of monthly values), (3) summer precipitation (sum of April to September monthly values), (4) winter precipitation (October to March), (5) potential yearly global radiation, (6) slope angle in degree, (7) topographic position (difference between the average elevation in a circular moving window applied to a 100m digital elevation model and the centre cell of the window (Zimmermann et al. 2007), (8) aspect value (ranging from 0(south) to 100(north), and (9) distance to running waters. These parameters were then used to explain the spatial distribution of a species. The statistical model calibration then evaluated, what parameter has predictive power and what parameter can be discarded or downweighted because it did not significantly add to explain the spatial distribution of a tree species.

Potential future climate was taken from six different RCMs (see previous chapter), providing a range of potential climate futures. The use of several RCM models is meant to provide the mean trend that can be expected from climate change impacts on trees but also some measure of uncertainty associated with the projection of these trends (Araujo \& New 2007, Thuiller et al. 2009). This was done because there is no consensus on which of the many climate models actually best describes the future faith of the climate. Rather, climatologists provide us with a range of models that are all considered equally likely to represent the climate future. Differences among climate models arise from divergent ways of parameterizing the complex dynamics of the global climate in such models. We therefore should not simply take one single model in order to assess the likely consequences of climate change on species range shifts. Our choice reflected differences in how much the different climate models simulated shifts in temperature and precipitation.

1 data from the National Inventory of Forests and Forest Carbon Pools -INFC 2005, National Forest Service of Italy - CFS 
Several statistical models were used, since the choice of a statistical model has been shown to significantly contribute to uncertainty in projections (Buisson et al. 2010). More specifically, we used the following statistical models: (1) Classification and regression trees (CART), (2) Flexible discriminant analysis (FDA), (3) Generalized linear models (GLM), (4) Generalized additive models (GAM), (5) Artificial neural networks (ANN), and (6) Generalized boosted regression trees (GBM). Therefore, given the use of six statistical models and six future climate model runs, we model 36 different possible futures per species and time period. This allows for assessing the projection uncertainty from both the variability in climate models and the variability originating from the choice of statistical methods. Naturally, the different models perform with different average accuracies among all species, with GBM and GAM often outperforming other models such as CART or GLM. However, when projecting models to future climates, it is not necessarily the currently best performing models that will yield predictions with lowest uncertainties! We know e.g. from projections to regions that are outside of the calibration region (but with similar climate) that GLMs outperformed GAMs, although within the calibration regions GAMs were always better than GLMs (Randin et al. 2006). Therefore, we chose to use all models as potentially equally likely to provide future ranges of our modelled tree species, and we only discarded those models, which didn't meet minimal criteria in a cross-validation test.

We optimized each statistical model following procedures described in Thuiller et al. (2003, 2009). Some models produce probabilities, while other generate directly presence/absence output. Where the statistical models generated probabilities, we used the kappa statistic (Cohen 1960) to select the best threshold to split the probability maps into maps of species presence and absence values. We therefore had one presence/absence map per climate model/ statistical model combination available. We then built ensembles of these model projections and classified these as follows: (1) a species is unlikely to find a suitable habitat if less than $30 \%$ of the projections indicated presence of a species; (2) a species is moderately likely, associated with high uncertainty, if $30-60 \%$ of the projections suggested that the species is there; (3) a species is most likely present with rather low uncertainty under projected climates if in $>60 \%$ of the 36 model projections presence of a species is simulated. Such a simple classification avoids an over-interpretation of the results from our modelling approach.

Figure 1 illustrates the potential future range shift in two species Fagus sylvatica L. (European beech) and Picea abies (L.) Karst. (Norway spruce) in eight panels, indicating the areas that are suitable for the two species under current and future climate conditions in three different time steps towards the end of the $21^{\text {st }}$ century. Both species are expected to lose much terrain at low altitudes, and will retract to higher altitudes following climate change. Currently, Norway spruce is planted at lower altitudes than it occurs naturally. Obviously, these lower altitudes are still within the fundamental niche of the species, and the simulated maps also capture the extended range of the species to lower altitudes under both current and future climates. However, compared to beech, it extends to higher altitudes, reaching treeline in many parts of the Alps. This is specifically visible for the simulations of the 2051-2080 time period, where for Norway spruce clearly higher elevations are projected to be suitable than for beech. For 
both species, larger parts at low altitudes become unsuitable, while the habitat suitability in large areas in Southern Germany are projected to be highly uncertain for both species. This uncertainty arises from highly contradicting projections by both climate and SDM model combinations.

More than 50 tree species have been simulated for the range of the Alps, and only two species are displayed here. A more complete set of species can be checked out and downloaded under a special website ${ }^{2}$. It becomes obvious that mostly the more drought-tolerant, sub-mediterranean species such as Quercus petraea and Quercus pubescens can be expected to become more abundant at lower altitudes throughout the Alps, while other species such as Acer pseudoplatanus, Tilia spp., Ulmus spp. or Abies alba are likely further reduced in their ranges similar to beech and spruce (Fig. 2). Species from Mediterranean regions such as Quercus ilex or Q. suber are expected to extend their ranges to the North, but these species will not (or only barely) reach the areas currently suitable for beech by the end of the $21^{\text {st }}$ century. Therefore, we can mostly expect sub-mediterranean, drought tolerant species to invade the spatial domain that is currently dominated by beech, namely: Q. petraea, Q. pubescens, Ostrya carpinifolia or Fraxinus ornus (see Fig.2). Different Pinus species are also expected to extend their ranges quite considerably. However, they will likely not extend to very fertile soils either, and some of the species like e.g. P. sylvestris might face indirect threats through insects and other pests, rather than direct threats from climate change alone. They may rather dominate at higher altitudes when summer precipitation is reduced, and larger parts of the Alps become considerably drier.

In fact, none of the individual SDM models is capable of projecting the effective fate of the different tree populations. The maps simply illustrate the habitat potential at certain time periods in the future. Species may still survive for quite a while at locations that become unsuitable. They will eventually face one or both of the following two threats: (1) physiological stress from a climate that they cannot tolerate, and (2) stronger competition by other, better adapted species or by antagonists such as insects, fungi, etc. that may profit in turn from a drier and/or hotter climate, and may infest trees that are less vigorous because of a combination of the two causes (1) and (2). Forest management can usually deal more or less well with the second type of threat if it is primarily due to changes in tree species competition. Dealing with changes in antagonists in forest management is more difficult, as the example of the Scots pine dieback in the Alps illustrates (Dobbertin et al. 2005, 2007, Bigler et al. 2006). Here, a rapid dieback at the lowest altitudes of the Scots pine distribution has been observed over the last 10 years, which is the area that is projected to eventually become unsuitable in the future from ensemble SDMs as well.

The presented SDM method has the advantage of rapidly indicating to managers and practitioners (1) the areas that become likely suitable soon, (2) the areas that are not expected to undergo significant changes, and (3) the areas that likely - and eventually - become unsuitable for maintaining viable tree populations naturally. The disadvantage of the method is that it cannot inform managers about the speed of the projected changes. Immigration into formerly unsuitable areas are not easy to predict, the invading species have to compete against the

2 http://www.wsl.ch/lud/manfred 

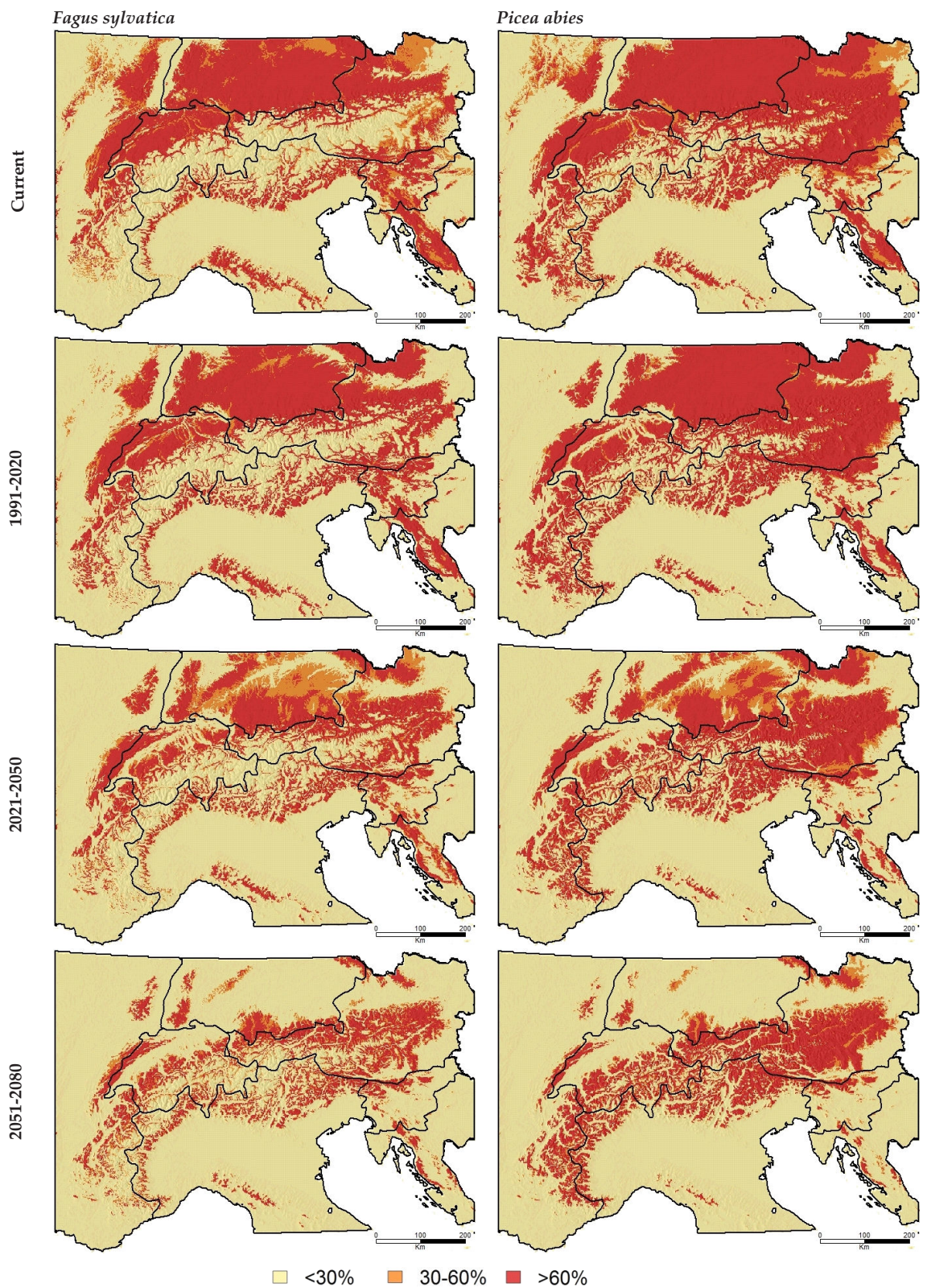

Figure 1. Projected future habitat suitability from ensemble SDM modeling of Fagus sylvatica and Picea abies for current and projected future climate in three time periods. Dark red colors represent high confidence of agreement of high habitat suitability from both six statistical and six climate models, while orange colors indicate high uncertainty in projected habitat suitability per time period. 

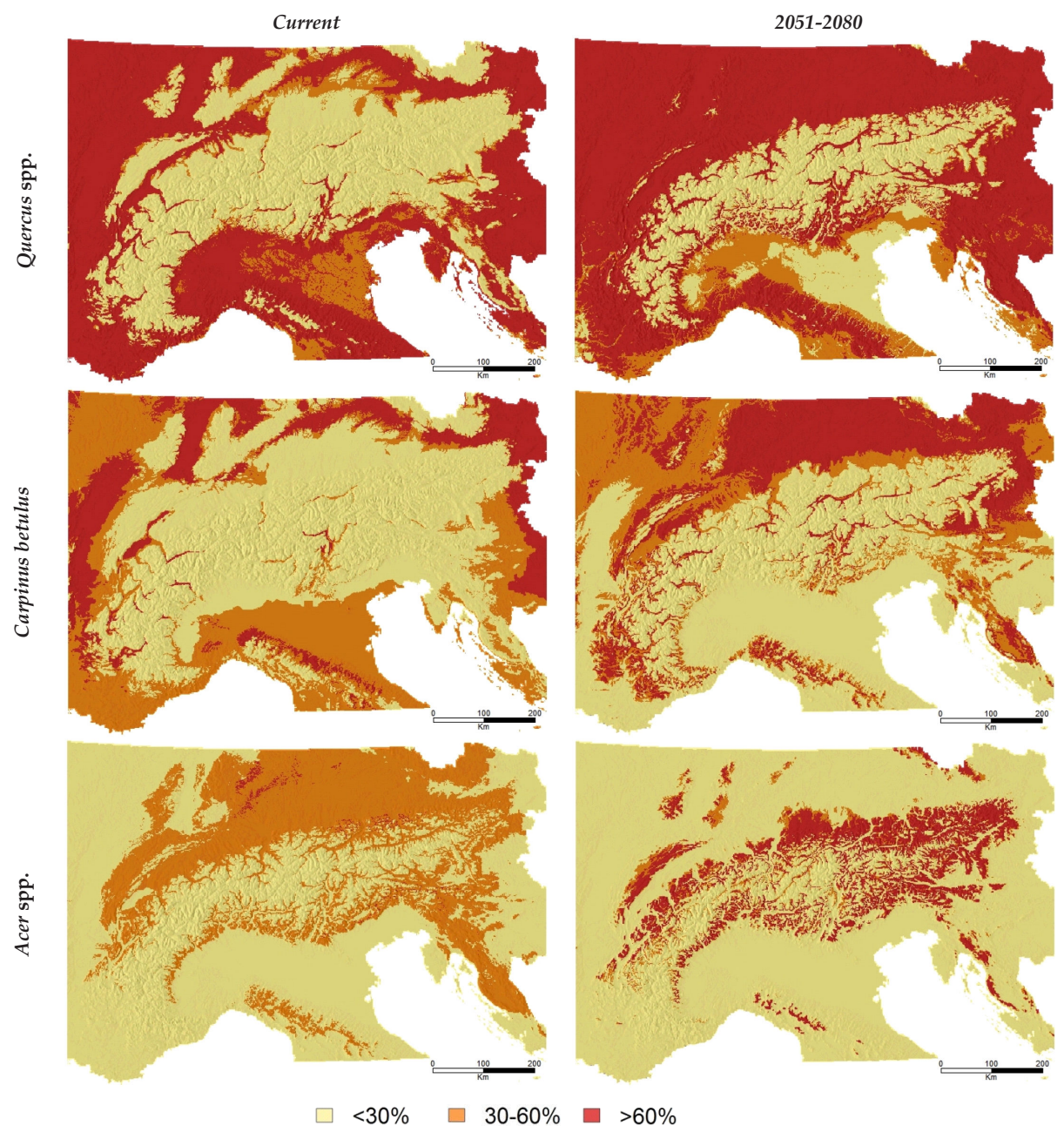

Figure 2. Projected future habitat suitability from ensemble SDM modeling of Quercus spp., Carpinus betulus, and Acer spp. for current and projected future climate in three time periods. Dark red colors represent high confidence of agreement of high habitat suitability from both six statistical and six climate models, while orange colors indicate high uncertainty in projected habitat suitability per time period. 
already inhabiting species, and the replacement process might take a long time. Even more difficult is to forecast at what rate tree species can no longer survive, or render financial benefits when planted or managed under slowly deteriorating climates. In fact, most species grow better under warmer climates. However, when at the same time increasing drought levels exceed species-specific thresholds, then trees might rapidly decline in growth or in maintaining viable populations. This illustrates, how difficult the forecasting of rear edge populations of a species range is under changing climate conditions. The model results need to be combined with careful observations.

SDMs can be improved, by adding more informative predictors, such as soil information or site productivity estimates. However, the difficulty to obtain these predictors with identical units and sufficient quality over large areas spanning the entire range of a species renders this type of model improvement often very difficult. And doing it only for smaller areas often results in models that do not span the capacity of the species well, because a smaller area that represents only a portion of the ecological niche of a species can be calibrated from data of such spatial domains. Yet, model improvements have to be sought constantly, and novel GIS techniques combined with the increasing availability of ecologically meaningful remote sensing data allows constant refinement of model predictors.

\section{Author details}

Niklaus E. Zimmermann ${ }^{1}$, Robert Jandl' ${ }^{2}$, Marc Hanewinkel ${ }^{1}$, Georges Kunstler ${ }^{4}$, Christian Kölling ${ }^{5}$, Patrizia Gasparini ${ }^{6}$, Andrej Breznikarr, Eliane S. Meier ${ }^{1}$, Signe Normand ${ }^{1}$, Ulrich Ulmer ${ }^{1}$, Thomas Gschwandtner ${ }^{2}$, Holger Veit ${ }^{3}$, Maria Naumann ${ }^{5}$, Wolfgang Falk ${ }^{5}$, Karl Mellert ${ }^{5}$, Maria Rizzo ${ }^{6}$, Mitja Skudnik ${ }^{8}$ and Achilleas Psomas ${ }^{1}$

1 Swiss Federal Research Institute WSL, Birmensdorf, Switzerland

2 Federal Research and Training Center for Forests, Natural Hazzards and Landscape BLW, Vienna, Austria

3 Forstliche Versuchs- und Forschungsanstalt Baden-Württemberg FVA, Freiburg, Germany

4 National Research Institute of Science and Technology for Environment and Agriculture IRSTEA, Grenoble, France

5 Bayerische Landesanstalt für Wald und Forstwirtschaft LWF, Freising, Germany

6 Consiglio per la Ricerca e la sperimentazione in Agricoltura - Unità di Ricerca per il Monitoraggio e la Pianificazione Forestale, CRA-MPF, Trento, Italy

7 Slovenia Forest Service, Lubljana, Slovenia

8 Slovenian Forestry Institute, Lubljana, Slovenia 


\section{References}

[1] Araujo, M. B, \& New, M. (2007). Ensemble forecasting of species distributions. Trends in Ecology \& Evolution , 22, 42-47.

[2] Bigler, C, Bräker, O. U, Bugmann, H, Dobbertin, M, \& Rigling, A. (2006). Drought as an inciting mortality factor in Scots pine stands of the Valais, Switzerland. Ecosystems , 9, 330-343.

[3] Dobbertin, M, et al. (2005). The decline of Pinus sylvestris L. forests in the swiss Rhone Valley- a result of drought stress? Phyton-Annales Rei Botanicae , 45, 153-156.

[4] Dobbertin, M, et al. (2007). Linking increasing drought stress to Scots pine mortality and bark beetle infestations. Thescientificworldjournal , 7, 231-239.

[5] Bugmann, H. (2001). A review of forest gap models. Climatic Change , 51, 259-305.

[6] Buisson, L, Thuiller, W, \& Casajus, N. Lek S \& Grenouillet G, (2010). Uncertainty in ensemble forecasting of species distribution. Global Change Biology , 16, 1145-1157.

[7] Chuine, I, \& Beaubien, E. G. (2001). Phenology is a major determinant of tree species range. Ecology Letters , 4, 500-510.

[8] Chuine, I. (2010). Why does phenology drive species distribution? Philosophical Transactions of the Royal Society B-Biological Sciences , 365, 3149-3160.

[9] Elith, J, et al. (2006). Novel methods improve prediction of species' distributions from occurrence data. Ecography , 29, 129-151.

[10] Guisan, A, \& Thuiller, W. (2005). Predicting species distribution: offering more than simple habitat models. Ecology Letters , 8, 993-1009.

[11] Guisan, A, \& Zimmermann, N. E. (2000). Predictive habitat distribution models in ecology. Ecological Modelling , 135, 147-186.

[12] Hickler, T, et al. (2012). Projecting the future distribution of European potential natural vegetation zones with a generalized, tree species-based dynamic vegetation model. Global Ecology and Biogeography , 21, 50-63.

[13] Huey, R. B, Kearney, M. R, \& Krockenberger, A. Holtum JAM, Jess M, Williams SE, (2012). Predicting organismal vulnerability to climate warming: roles of behaviour, physiology and adaptation. Philosophical Transactions of the Royal Society B-Biological Sciences , 367, 1665-1679.

[14] Hutchinson, G. E. (1957). Concluding remarks. Cold Spring Harbour Symposium on Quantitative Biology , 22, 415-427.

[15] Lischke, H, Zimmermann, N. E, Bolliger, J, Rickebusch, S, \& Löffler, T. J. (2006). TreeMig: A forest-landscape model for simulating spatio-temporal patterns from stand to landscape scale. Ecological Modelling , 199, 409-420. 
[16] Meier, E. S, Edwards, T. C, Kienast, F, Dobbertin, M, \& Zimmermann, N. E. (2011). Co-occurrence patterns of trees along macro-climatic gradients and their potential influence on the present and future distribution of Fagus sylvatica L. Journal of Biogeography , 38, 371-382.

[17] Meier, E. S, et al. (2010). Biotic and abiotic variables show little redundancy in explaining tree species distributions. Ecography , 33, 1038-1048.

[18] Meier, E. S, Lischke, H, Schmatz, D. R, \& Zimmermann, N. E. (2012). Climate, competition and connectivity affect future migration and ranges of European trees. Global Ecology and Biogeography , 21, 164-178.

[19] Randin, C. F, Dirnböck, T, Dullinger, S, \& Zimmermann, N. E. Zappa M \& Guisan A, (2006). Are species distribution models transferable in space? Journal of Biogeography , 33, 1689-1703.

[20] Schumacher, S, \& Bugmann, H. (2006). The relative importance of climatic effects, wildfires and management for future forest landscape dynamics in the Swiss Alps. Global Change Biology , 12, 1435-1450.

[21] Schurr, F. M, Pagel, J, Cabral, J. S, Groeneveld, J, Bykova, O, Hara, O, Kissling, R. B, Linder, W. D, Midgley, H. P, Schröder, G. F, Singer, B, \& Zimmermann, A. NE, in press. How to understand species niches and range dynamics: a demographic research agenda for biogeography. Journal of Biogeography.

[22] Sitch, S, et al. (2003). Evaluation of ecosystem dynamics, plant geography and terrestrial carbon cycling in the LPJ dynamic global vegetation model. Global Change Biology , 9, 161-185.

[23] Thornton, P. E, et al. (2002). Modeling and measuring the effects of disturbance history and climate on carbon and water budgets in evergreen needleleaf forests. Agricultural and Forest Meteorology , 113, 185-222.

[24] Thuiller, W. (2003). BIOMOD- optimizing predictions of species distributions and projecting potential future shifts under global change. Global Change Biology , 9, 1353-1362.

[25] Thuiller, W, Lafourcade, B, Engler, R, \& Araujo, M. B. (2009). BIOMOD- a platform for ensemble forecasting of species distributions. Ecography , 32, 369-373.

[26] Woodward, F. I. (1987). Climate and plant distribution. Cambridge University Press, Cambridge

[27] Zimmermann, N. E, Edwards, T. C, Moisen, G. G, Frescino, T. S, \& Blackard, J. A. (2007). Remote sensing-based predictors improve distribution models of rare, early successional and broadleaf tree species in Utah. Journal of Applied Ecology , 44, 1057-1067. 
[28] Zimmermann, N. E, et al. (2009). Climatic extremes improve predictions of spatial patterns of tree species. Proceedings of the National Academy of Sciences of the United States of America , 106, 19723-19728.

[29] Zimmermann, N. E, Edwards, T. C, Graham, C. H, Pearman, P. B, \& Svenning, J. C. (2010). New trends in species distribution modelling. Ecography , 33, 985-989. 